\title{
Bilirubin levels as an independent predictor of myocarditis in patients with COVID-19
}

\author{
Muharrem Said Cosgun* ${ }^{*}$
}

\begin{abstract}
Background: Myocardial damage worsens the clinical course and prognosis of coronavirus disease 2019 (COVID19) patients. High total bilirubin levels have been associated with a poor prognosis in COVID-19. This study aimed to investigate the predictive value of the total bilirubin level, a marker of heme oxygenase-1 enzyme activity, in determining myocarditis in patients with COVID-19.

Results: A total of 190 patients diagnosed with COVID-19 were enrolled in the study. The patients were divided into two groups based on their troponin positivity. The study group $(n=95)$ consisted of patients with high troponin, and the control group $(n=95)$ consisted of patients without high troponin levels. The D-dimer (727 [572-995] vs. 591 [440-790], $p=0.001)$, C-reactive protein (CRP) (30.0 [10-48] vs. 10.3 [5.8-15.9], $p<0.001)$, and total bilirubin (9.5 [8.2-12.1] vs. 7.0 [5.3-8.0], $p<0.001)$ levels were significantly higher in the study group. In multivariate analysis, CRP (odds ratio [OR]: 1.103; 95\% confidence interval [Cl]: 1.060-1.148; $p<0.001)$ and total bilirubin (OR: 1.612; 95\% Cl: $1.330-1.954 ; p<0.001$ ) levels were independent predictors of myocarditis in COVID-19.
\end{abstract}

Conclusions: Total bilirubin levels can be used as an early predictor of myocarditis in COVID-19 and can contribute to therapy management.

Keywords: Bilirubin, Myocarditis, COVID-19, Microthrombi

\section{Background}

Human coronavirus $(\mathrm{CoV})$ infections were considered to cause mild respiratory disease in the twentieth century [1]. However, this has changed in the first 20 years of the new century, with three outbreaks of CoVs causing significant mortality and morbidity [2]. The most important common feature of these outbreaks is that the infection becomes systemic and causes acute respiratory distress syndrome (ARDS) through autoimmune mechanisms [3, 4]. The third pandemic, called coronavirus disease 2019 (COVID-19) and caused by the severe acute respiratory syndrome (SARS)-CoV-2, started in late 2019 and continues at the time of this writing, with transmission rates higher than those of both previous $\mathrm{CoV}$ outbreaks $[5,6]$.

\footnotetext{
*Correspondence: drsaidcosgun2009@hotmail.com
}

Department of Cardiology, Mengucek Gazi Education and Research Hospital, Medical Faculty, Erzincan Binali Yildirim University, Erzincan, Turkey
Existing cardiovascular comorbidities increase sensitivity to COVID-19. Moreover, COVID-19 can aggravate the underlying cardiovascular disease and lead to cardiac complications [5]. Although the infection is mild in most people, if the severe and critical form of the disease develops, the risk of multi-organ damage increases, including damage to the heart and death $[7,8]$. Therefore, early diagnosis of multi-organ damage, especially heart injury, is essential. Healthcare professionals have naturally focused on lung injury, while other organ involvement, such as heart damage, has remained in the background. Troponin is the best indicator of myocardial injury due to COVID-19, as in all types of myocardial damage [9]. The available literature shows that myocardial damage worsens the clinical course and prognosis [10, 11]. For example, one study showed that more than half of the patients who died had an acute myocardial injury and that the extrapulmonary organ most commonly affected by COVID-19 was the heart [12]. 
Numerous reviews and meta-analyses have indicated that serum bilirubin levels are significantly high in patients with severe COVID-19 symptoms, such as pneumonia, ARDS, multi-organ damage, and septic shock [13-17]. Bilirubin is a marker of heme oxygenase-1 (HO1) enzyme activity and is the end-product of heme reduction [18]. Free heme, the precursor of bilirubin, has been blamed in COVID-19 pathogenesis through its triggering of inflammatory processes, vascular permeabilization, and thrombosis [19]. However, total bilirubin levels are associated with both troponin increase and intracoronary thrombus burden in patients with acute coronary syndrome characterized by myocardial damage $[20,21]$.

\section{Aim}

No studies in the current literature have examined the relationship between myocardial damage and total bilirubin levels in COVID-19 patients. Therefore, the purpose of this study was to investigate the relationship between total bilirubin levels, a marker of heme oxygenase-1 enzyme activity, and myocarditis in COVID-19.

\section{Methods \\ Study population}

This study was a uni-center, retrospective, descriptive, and observational study using cross-sectional data collected from the COVID-19 patients. One hundred and ninety patients whose diagnosis of COVID-19 was confirmed by reverse transcription-polymerase chain reaction (RT-PCR) were included in the study. The patients were allocated into two groups based on their troponin positivity. The consecutive patients in the study group had chest pain and elevated troponin with normal coronary angiography $(n=95)$. The control group consisted of 95 consecutive patients with similar demographic and clinical characteristics but normal troponin values. The following were exclusion criteria: (1) atherosclerotic lesion(s) in coronary angiography; (2) previous coronary artery disease; (3) referral to intensive care units due to severe and critical illness; (4) asymptomatic COVID-19 disease; (5) severe liver and kidney disease; (6) known malignancy or systemic inflammatory disease; (7) presence of anemia (hemoglobin $<13$ and $<12 \mathrm{~g} / \mathrm{dL}$ for men and women, respectively) or a history of blood transfusions in the last 90 days; (8) a negative RT-PCR test for COVID-19.

\section{Laboratory analysis}

Electrocardiography measurements and troponin I levels were repeated at 4- to 6-h intervals in patients with chest pain. Serum bilirubin levels were analyzed with an autoanalyzer (AU2700 Plus analyzer, Beckman Coulter, Tokyo, Japan). Complete blood counts (hemoglobin, white blood cell, and platelet) and glucose, creatinine, creatine kinase myocardial band (CK-MB), D-dimer, and C-reactive protein (CRP) levels, and liver function tests (aspartate aminotransferase [AST] and alanine aminotransferase [ALT]) were studied in blood samples taken during admission.

\section{Clinical definitions}

Age, gender, smoking habits, the treatment regimen for COVID-19, previous medications, and diseases were registered for both groups. The diagnoses of hypertension (HT), hyperlipidemia (HL), and diabetes mellitus (DM) were based on the previous history. The severity of COVID-19 pneumonia was defined per the guidelines of Diagnosis and Treatment of Pneumonia Caused by SARS-CoV-2- (1) mild: asymptomatic and radiologically normal patients; (2) moderate: symptomatic and patients with pneumonia on computed tomography; (3) severe: patients with fingertip oxygen saturation $\leq 93 \%$ and respiratory rate $>30 / \mathrm{min}$ while breathing room air; and (4) critical: patients requiring intensive care referral [22]. A level of troponin I exceeding the 99th-percentile upper reference limit was accepted as myocardial damage [11, 23].

\section{Statistical analysis}

Categorical variables were compared with the chi-square test and shown as percentages (\%). For the analysis of continuous variables, their distribution was evaluated using the One-Sample Kolmogorov-Smirnov test. The $t$ test was used if the continuous variables between the two groups were normally distributed, and the MannWhitney $U$ test was used if they were not. Continuous variables were shown as mean \pm standard deviation if they were normally distributed, and as median (1st-3rd quartiles) if not. Independent predictors of myocarditis were determined by multivariate logistic regression analysis and presented with $95 \%$ confidence interval (CI) and odds ratio (OR). The cut-off value of the total bilirubin level was analyzed by the receiver-operating characteristic (ROC) curve. Statistical significance value was considered as $p<0.05$. All data obtained were transferred to SPSS version 22 and analyzed (IBM, SPSS Statistics, USA).

\section{Results}

The study group $(n=95$; mean age $64.3+9.8$ years; $73.7 \%$ male) consisted of troponin positive patients, while the control group $(n=95$; mean age $62.3+9.3$ years; $69.5 \%$ male) consisted of troponin negative patients. Table 1 shows a comparison of the baseline demographic and clinical characteristics and laboratory results. No significant difference was noted 
Table 1 Baseline demographic and clinical characteristics and laboratory results according to the presence of myocarditis

\begin{tabular}{|c|c|c|c|}
\hline Variables & Troponin positive $(n=95)$ & Troponin negative $(n=95)$ & $p$ value \\
\hline Age, years & $64.3 \pm 9.8$ & $62.3 \pm 9.3$ & 0.158 \\
\hline Gender, male, $n(\%)$ & $70(73.7)$ & $66(69.5)$ & 0.52 \\
\hline Smoking habits, $n(\%)$ & $22(23.2)$ & $20(21.1)$ & 0.727 \\
\hline Hypertension, $n(\%)$ & $35(36.8)$ & $31(32.6)$ & 0.542 \\
\hline Hyperlipidemia, n (\%) & $23(24.2)$ & $21(22.1)$ & 0.731 \\
\hline Diabetes mellitus, $n$ (\%) & $16(16.8)$ & $14(14.7)$ & 0.691 \\
\hline Hemoglobin, g/dL & $13.5 \pm 1.4$ & $13.8 \pm 1.1$ & 0.122 \\
\hline White blood cell count, $\times 10^{9} / \mathrm{L}$ & $5.9 \pm 2.1$ & $5.6 \pm 2.1$ & 0.157 \\
\hline Platelet count, $\times 10^{9} / \mathrm{L}$ & $213(158-235)$ & $219(184-262)$ & 0.081 \\
\hline Glucose, mg/dL & $103(91-123)$ & $100(95-115)$ & 0.465 \\
\hline Creatinine, $\mathrm{mg} / \mathrm{dL}$ & $0.82 \pm 0.21$ & $0.81 \pm 0.18$ & 0.804 \\
\hline Aspartate aminotransferase, $U / L$ & $30(21-36)$ & $29(23-36)$ & 0.824 \\
\hline Alanine aminotransferase, $\mathrm{U} / \mathrm{L}$ & $22(17-29)$ & $25(17-41)$ & 0.318 \\
\hline D-dimer, $\mu \mathrm{g} / \mathrm{L}$ & 727 (572-995) & $591(440-790)$ & 0.001 \\
\hline C-reactive protein, $\mathrm{mg} / \mathrm{L}$ & $30.0(10-48)$ & $10.3(5.8-15.9)$ & $<0.001$ \\
\hline CK-MB* ${ }^{*}, \mathrm{U} / \mathrm{L}$ & $19.2(13.7-27.4)$ & $17.7(14.1-20.2)$ & 0.077 \\
\hline Total bilirubin, $\mu \mathrm{mol} / \mathrm{L}$ & $9.5(8.2-12.1)$ & $7.0(5.3-8.0)$ & $<0.001$ \\
\hline \multicolumn{4}{|l|}{ Previous medications } \\
\hline $\operatorname{RAASB}^{\dagger}, n(\%)$ & $22(23.2)$ & $24(25.3)$ & 0.735 \\
\hline Calcium channel blockers, $n(\%)$ & $14(14.7)$ & $11(11.6)$ & 0.52 \\
\hline Beta-blockers, n (\%) & $6(6.3)$ & $7(7.4)$ & 0.774 \\
\hline Statin, $n(\%)$ & $10(10.5)$ & $14(14.7)$ & 0.382 \\
\hline Antiaggregant, $n(\%)$ & $9(9.5)$ & $11(11.6)$ & 0.636 \\
\hline \multicolumn{4}{|l|}{ Treatment regimen } \\
\hline Antiviral, $n(\%)$ & $86(90.5)$ & $83(87.4)$ & 0.488 \\
\hline Antibiotic, $n(\%)$ & $67(70.5)$ & $65(68.4)$ & 0.753 \\
\hline Hydroxychloroquine, $n(\%)$ & $25(26.3)$ & $22(23.2)$ & 0.614 \\
\hline $\mathrm{LMWH}^{\ddagger}, n(\%)$ & $89(93.7)$ & $87(91.6)$ & 0.579 \\
\hline Corticosteroid, $n(\%)$ & $48(50.5)$ & $44(46.3)$ & 0.561 \\
\hline
\end{tabular}

*Creatine kinase myocardial band

${ }^{\dagger}$ Renin-angiotensin-aldosterone system blockers

${ }^{\ddagger}$ Low-molecular-weight heparin

Bold defines statistically significant values

between groups in terms of age, gender, smoking habits, or previous history of HT, HL, and DM. Complete blood counts (hemoglobin, white blood cells, and platelets) and glucose, creatinine, AST, ALT, and CK-MB levels in the blood samples taken at the admission did not differ significantly between the groups. Previous medications, renin-angiotensin-aldosterone system blockers, calcium channel blockers, beta-blockers, statin, and antiaggregant were not meaningfully different between the groups. Antiviral, antibiotic, hydroxychloroquine, low molecular weight heparin, and corticosteroid treatment regimens for COVID-19 were similar between the groups. The D-dimer (727 [572-995] vs. 591 [440-790], $p=0.001)$, CRP (30.0 [10-48] vs. 10.3 [5.8-15.9], $p<0.001)$, and total bilirubin (9.5 [8.2-12.1] vs. $7.0[5.3-8.0], p<0.001)$ levels were meaningfully higher in the troponin-positive patients. The distribution of total bilirubin levels in the negative and positive troponin groups is shown in Fig. 1.

Independent predictors of myocarditis were determined by multivariate logistic regression analysis. The CRP (OR: 1.103; 95\% CI: 1.060-1.148; $p<0.001$ ) and total bilirubin (OR: 1.612; 95\% CI: 1.330-1.954; $p<0.001)$ levels were independent predictors of myocarditis in COVID-19 (Table 2). The ROC analysis revealed a cut-off value of total bilirubin for myocarditis of $8.48 \mu \mathrm{mol} / \mathrm{L}$, with a sensitivity of $71.6 \%$ and a specificity of $77.9 \%$ (area under curve, 0.773 ; $95 \% \mathrm{CI}$, $0.704-0.841 ; p<0.001 ;$ Fig. 2 ). 


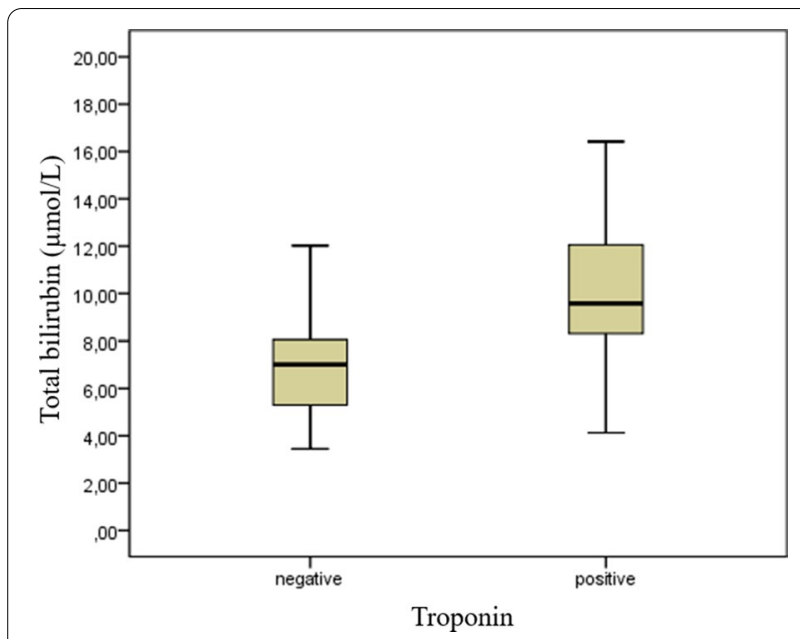

Fig. 1 Total bilirubin levels between negative and positive troponin groups

Table 2 Multivariate logistic regression analysis to assess predictors of myocarditis

\begin{tabular}{lcc}
\hline Variables & Odds ratio $\left(\mathbf{9 5 \%} \mathrm{Cl}^{*}\right)$ & $\boldsymbol{p}$ value \\
\hline $\mathrm{CK}-\mathrm{MB}^{\dagger}, \mathrm{U} / \mathrm{L}$ & $1.002(0.967-1.038)$ & 0.921 \\
C-reactive protein, $\mathrm{mg} / \mathrm{L}$ & $1.103(1.060-1.148)$ & $<0.001$ \\
D-dimer, $\mu \mathrm{g} / \mathrm{L}$ & $1.001(1.000-1.002)$ & 0.108 \\
Platelet counts, $\times 10^{9} / \mathrm{L}$ & $1.000(0.995-1.005)$ & 0.982 \\
Total bilirubin, $\mu \mathrm{mol} / \mathrm{L}$ & $1.612(1.330-1.954)$ & $<\mathbf{0 . 0 0 1}$
\end{tabular}

*Confidence interval

${ }^{\dagger}$ Creatine kinase myocardial band

Bold defines statistically significant values

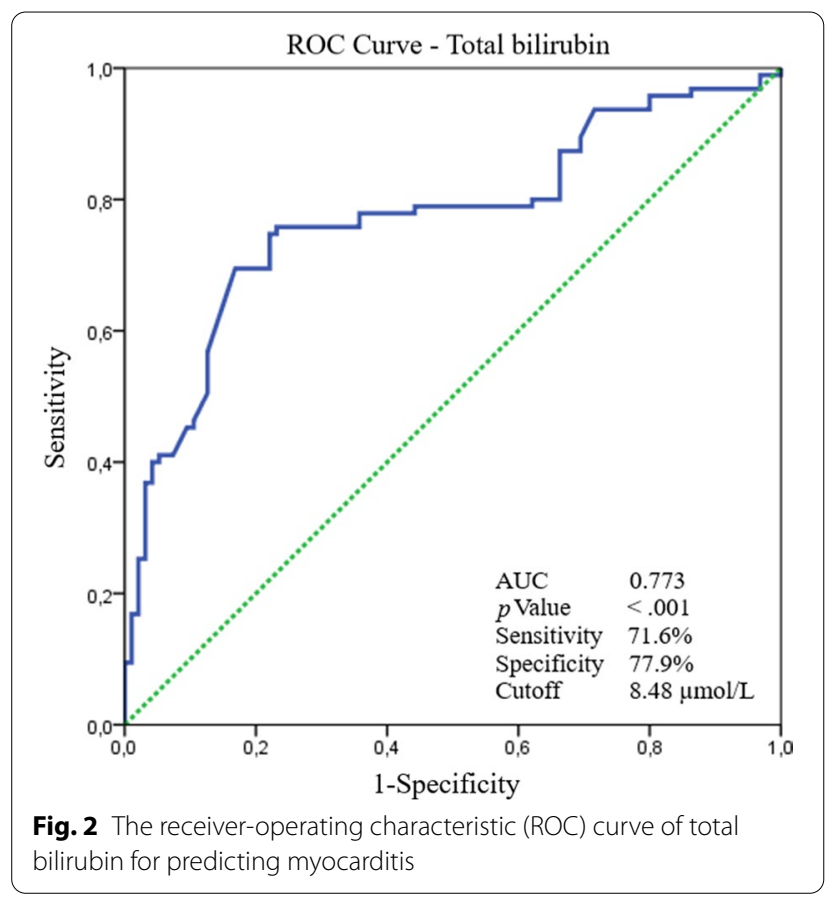

\section{Discussion}

The results of our study showed that the total bilirubin levels, which are an indicator of HO-1 enzyme activity, and the CRP levels were independent predictors of myocarditis in COVID-19 patients. In addition, high D-dimer levels were correlated with myocarditis.

The SARS-CoV-2 enters host cells via endocytosis mediated by angiotensin-converting enzyme 2 (ACE-2) receptors. The virus causes ARDS due to ACE- 2 receptors, commonly expressed in Type 2 pneumocytes of the lungs [24]. The same mechanism frequently targets the myocytes in the heart and enterocytes in the intestines secondary to the lungs [25]. Possible mechanisms proposed to explain the pathogenesis of myocardial injury in COVID-19 have included direct damage related to viral myocarditis, a systemic hyperinflammatory response caused by a cytokine storm, a myocardial oxygen demand-supply mismatch due to hypoxemia, down-regulation of ACE-2 receptors, systemic virusinduced endothelialitis, type 1-2 myocardial infarction, and iatrogenic effects due to corticosteroid and hydroxychloroquine use [26]. Recent autopsy-based studies have also implicated microthrombi as responsible for the etiopathogenesis of myocardial injury [27, 28]. A large-scale meta-analysis supporting this information highlighted the importance of anticoagulant therapy in COVID-19 due to the relationship between $\mathrm{D}$-dimer and thrombosis [29].

Bilirubin, which has endogenous anti-oxidant and anti-inflammatory effects, is the end-product of heme catabolism. The HO enzyme catalyzes the reduction in heme groups and converts heme to carbon monoxide, ferrous iron, and biliverdin. Biliverdin is then rapidly degraded to bilirubin and excreted in the urine, while the ferrous iron is inactivated by binding to ferritin [30, 31]. Heme is highly cytotoxic due to its reactive nature, but bilirubin, the final product formed after degradation by the $\mathrm{HO}$ enzyme, is a scavenger of reactive oxygen species [32]. Under normal conditions, bilirubin has been shown to protect against adverse cardiovascular events due to its anti-oxidant effects [33-37]. The $\mathrm{HO}$ enzyme has three isoforms, with $\mathrm{HO}-1$ being the rate-limiting enzyme in the heme degradation pathway. The enzyme activity of HO-1 is significantly upregulated in response to acute stress. High levels of heme and HO-1 have been detected in severe and hypoxic COVID-19 patients [38]. Based on this, HO-1 pathway immunomodulation has been viewed as a potential therapeutic strategy against COVID-19 and associated complications [39]. Conversely, the other isoforms (HO-2 and $\mathrm{HO}-3$ ) are structural and their expression is not affected by external factors such as stress [18]. We evaluated serum bilirubin levels in the present study 
rather than HO-1 activity, because this analysis is inexpensive, easily accessible, and frequently used in daily practice. Okuhara et al. previously demonstrated the relationship between HO-1 enzyme activity and serum bilirubin levels in myocardial damage [40].

High serum bilirubin levels have been correlated with the burden of atherosclerosis and thrombus, low thrombolysis in myocardial infarction (TIMI) flow grade, and high in-hospital major adverse cardiac events (MACEs) in patients with ST-segment elevation myocardial infarction (STEMI) [21, 41, 42]. A correlation between total bilirubin levels and troponin has also been reported in non-STEMI patients [20]. Hamur et al. confirmed that total bilirubin levels are correlated with thrombus burden in patients with STEMI and that bilirubin is an independent predictor of high thrombus burden. Based on this, they argued that advanced anticoagulant therapy could be used in patients with elevated serum bilirubin levels and could contribute to the management of intracoronary thrombus burden [21].

As explained above, microthrombi plays an important role in COVID-19 pathogenesis and associated myocardial damage, and serum bilirubin levels are correlated with thrombus burden in stress conditions, such as acute coronary syndrome $[21,27,28]$. We designed our study based on these observations, and we determined that total bilirubin levels are an independent predictor of myocarditis in COVID-19. In addition, the CRP levels were confirmed as an independent predictor of myocarditis in COVID-19, along with bilirubin. CRP is a strong indicator of inflammation, and high serum levels are a strong and independent predictor of cardiovascular adverse events [43]. We also found significantly higher D-dimer levels in COVID-19 patients with myocarditis, consistent with previous studies. D-dimer is an indirect marker of coagulation turnover and serves as an indicator of intravascular thrombosis [44]. Previous studies have shown an association between CRP and D-dimer levels and myocardial injury in COVID-19 [45, 46].

\section{Study limitations}

The most important limitation of this study is that we did not directly evaluate the HO- 1 enzyme activity. Myocardial injury was diagnosed only by troponin elevation, and coronary angiography showed patency of epicardial coronary arteries. The absence of cardiac magnetic resonance or endomyocardial biopsy for further examination is another significant limitation. However, the pressure of the pandemic on health care may explain this situation. Another limitation is the small number of patients included in the study. It was also a retrospective study; therefore, we did not analyze long-term events.

\section{Conclusions}

The total bilirubin level can be used as an early predictor of myocarditis in COVID-19, as it is inexpensive to measure, easily accessible, and frequently used in daily practice. Thus, it can serve as a guide for optimal anticoagulant therapy and contribute to managing high-risk patients with COVID-19. However, its potential needs to be confirmed by further clinical studies. The new point of this study is that total bilirubin level, a marker of heme oxygenase- 1 enzyme activity, can predict heart damage in patients with COVID-19.

\section{Abbreviations \\ ACE-2: Angiotensin-converting enzyme 2; ALT: Alanine aminotransferase; ARDS: Acute respiratory distress syndrome; AST: Aspartate aminotransferase; $\mathrm{Cl}$ : Confidence interval; CK-MB: Creatine kinase myocardial band; CoV: Coro- navirus; COVID-19: Coronavirus disease 2019; CRP: C-reactive protein; DM: Dia- betes mellitus; HL: Hyperlipidemia ; HO: Heme oxygenase; HT: Hypertension; MACEs: Major adverse cardiac events; OR: Odds ratio; ROC: Receiver-operating characteristic; RT-PCR: Reverse transcription-polymerase chain reaction; SARS: Severe acute respiratory syndrome; STEMI: ST-segment elevation myocardial infarction; TIMI: Thrombolysis in myocardial infarction.}

\section{Acknowledgements \\ Not applicable.}

\section{Authors' contributions}

MSC: Conceived the idea, planned the study design, organized data acquisition, and interpretation, and performed statistical data analysis and writing.

\section{Funding}

None. This research did not receive any specific grant from funding agencies in the public, commercial, or not-for-profit sectors.

\section{Availability of data and materials}

The datasets used and/or analyzed during the current study are available from the corresponding author on reasonable request.

\section{Declarations}

\section{Ethics approval and consent to participate}

The study protocol conforms to the Institutional and National Human Experimentation Committee and the Declaration of Helsinki of 1975, revised in 2008. The Erzincan Binali Yildirim University Ethics Committee approved our study (date: 07/07/2020, meeting no: 07, and protocol number: 06) and the Turkish Ministry of Health Research Board (2020-05-11T11_23_01). An obtained written informed consent form was from each participant.

Consent for publication

Consent was obtained from each participant.

Competing interests

The author declares that he has no competing interests.

Received: 23 June 2021 Accepted: 13 December 2021 Published online: 20 December 2021

References

1. De Wilde AH et al (2017) Host factors in coronavirus replication. In: Tripp RA, MarkTompkins S (eds) Roles of host gene and non-coding RNA expression in virus infection. Springer, pp 1-42 
2. Tort FL, Castells M, Cristina J (2020) A comprehensive analysis of genome composition and codon usage patterns of emerging coronaviruses. Virus Res 283:197976

3. Cui J, Li F, Shi ZL (2019) Origin and evolution of pathogenic coronaviruses. Nat Rev Microbiol 17(3):181-192

4. Clerkin KJ et al (2020) COVID-19 and cardiovascular disease. Circulation 141(20):1648-1655

5. Bansal M (2020) Cardiovascular disease and COVID-19. Diabetes Metab Syndr 14(3):247-250

6. Ye Z-W et al (2020) Zoonotic origins of human coronaviruses. Int J Biol Sci 16(10):1686

7. Alhazzani W, Du B (2020) Surviving Sepsis Campaign: guidelines on the management of critically ill adults with Coronavirus Disease 2019 (COVID19). Intensive Care Med 46:1-34

8. Yang $X$ et al (2020) Clinical course and outcomes of critically ill patients with SARS-CoV-2 pneumonia in Wuhan, China: a single-centered, retrospective, observational study. Lancet Respir Med 8(5):475-481

9. Deng P et al (2020) The diagnostic and prognostic role of myocardial injury biomarkers in hospitalized patients with COVID-19. Clin Chim Acta 510:186-190

10. Ferrari R, Di Pasquale G, Rapezzi C (2020) Commentary: what is the relationship between Covid-19 and cardiovascular disease? Int J Cardiol 310:167

11. Yang C et al (2021) Myocardial injury and risk factors for mortality in patients with COVID-19 pneumonia. Int J Cardiol 326:230-236

12. Shi S et al (2020) Association of cardiac injury with mortality in hospitalized patients with COVID-19 in Wuhan. JAMA Cardiol. 5:8072-8810

13. Bao J et al (2020) Comparative analysis of laboratory indexes of severe and non-severe patients infected with COVID-19. Clin Chim Acta 509:180-194

14. Danwang C et al (2020) A meta-analysis of potential biomarkers associated with severity of coronavirus disease 2019 (COVID-19). Biomark Res $8(1): 1-13$

15. Ghahramani S et al (2020) Laboratory features of severe vs. non-severe COVID-19 patients in Asian populations: a systematic review and metaanalysis. Eur J Med Res 25(1):1-10

16 Izcovich A et al (2020) Prognostic factors for severity and mortality in patients infected with COVID-19: a systematic review. PLoS ONE 15(11):e0241955

17. Paliogiannis P, Zinellu A (2020) Bilirubin levels in patients with mild and severe Covid-19: a pooled analysis. Liver Int 40(7):1787-1788

18. Siow RCM, Sato H, Mann GE (1999) Heme oxygenase-carbon monoxide signalling pathway in atherosclerosis: anti-atherogenic actions of bilirubin and carbon monoxide? Cardiovasc Res 41(2):385-394

19 Wagener $\mathrm{F}$ et al (2020) Targeting the heme-heme oxygenase system to prevent severe complications following COVID-19 infections. Antioxidants 9(6):540

20. Ozturk M et al (2017) The role of serum bilirubin levels in predicting troponin positivity in non-ST-segment elevation acute coronary syndrome. Angiology 68(5):414-418

21. Hamur $\mathrm{H}$ et al (2016) Bilirubin levels and thrombus burden in patients with ST-segment elevation myocardial infarction. Angiology 67(6):565-570

22. National Health Commission (2020) Diagnosis and treatment protocol for novel coronavirus pneumonia (trial version 7). Chin Med J (Engl) 133(9):1087-1095

23. Caforio AL et al (2013) Current state of knowledge on aetiology, diagnosis, management, and therapy of myocarditis: a position statement of the European Society of Cardiology Working Group on Myocardial and Pericardial Diseases. Eur Heart J 34(33):2636-2648

24. Zhu N et al (2020) A novel coronavirus from patients with pneumonia in China, 2019. N Engl J Med 382(8):727-733

25. Xu H et al (2020) High expression of ACE2 receptor of 2019-nCoV on the epithelial cells of oral mucosa. Int J Oral Sci 12(1):1-5

26. Ozieranski K et al (2021) Clinically suspected myocarditis in the course of severe acute respiratory syndrome novel coronavirus-2 infection: fact or fiction? J Card Fail 27(1):92-96

27. Bois MC et al (2021) COVID-19-associated nonocclusive fibrin microthrombi in the heart. Circulation 143(3):230-243

28 Pellegrini D et al (2021) Microthrombi as a major cause of cardiac injury in COVID-19: a pathologic study. Circulation 143:1031-1042
29. McBane RD 2nd et al (2020) Anticoagulation in COVID-19: a systematic review, meta-analysis, and rapid guidance from Mayo Clinic. Mayo Clin Proc 95(11):2467-2486

30. Tenhunen R, Marver HS, Schmid R (1968) The enzymatic conversion of heme to bilirubin by microsomal heme oxygenase. Proc Natl Acad Sci USA 61(2):748-755

31. Tenhunen R, Marver HS, Schmid R (1969) Microsomal heme oxygenase: characterization of the enzyme. J Biol Chem 244(23):6388-6394

32 Maines MD, Trakshel GM, Kutty RK (1986) Characterization of two constitutive forms of rat liver microsomal heme oxygenase. Only one molecular species of the enzyme is inducible. J Biol Chem 261(1):411-419

33. Wang $\mathrm{H}-\mathrm{Y}$ et al (2012) Serum bilirubin level is negatively correlated with disease progression of peripheral arterial disease: an observational cohort study. Angiology 63(4):248-253

34. Kalkan GY et al (2014) Serum bilirubin level and aortic intima-media thickness in patients without clinical manifestation of atherosclerotic cardiovascular disease. Angiology 65(4):308-312

35. Hamur $\mathrm{H}$ et al (2016) Total bilirubin levels predict subclinical atherosclerosis in patients with prediabetes. Angiology 67(10):909-915

36. Hamur $\mathrm{H}$ et al (2017) Determinants of chronic total occlusion in patients with peripheral arterial occlusive disease. Angiology 68(2):151-158

37. Lan $Y$ et al (2020) The relationship between serum bilirubin levels and peripheral arterial disease and gender difference in patients with hypertension: BEST study. Angiology 71(4):340-348

38. Su WL et al (2021) Desaturation and heme elevation during COVID-19 infection: a potential prognostic factor of heme oxygenase-1. J Microbiol Immunol Infect 54(1):113-116

39. Singh $D$, Wasan $H$, Reeta KH (2020) Heme oxygenase-1 modulation: a potential therapeutic target for COVID-19 and associated complications. Free Radic Biol Med 161:263-271

40. Okuhara K et al (2010) Change in bilirubin level following acute myocardial infarction is an index for heme oxygenase activation. South Med J 103(9):876-881

41. Sahin $O$ et al (2013) Bilirubin levels and the burden of coronary atherosclerosis in patients with STEMI. Angiology 64(3):200-204

42. Celik T et al (2014) Does serum bilirubin level on admission predict TIMI flow grade and in-hospital MACE in patients with STEMI undergoing primary PCl. Angiology 65(3):198-204

43. Sproston NR, Ashworth JJ (2018) Role of C-reactive protein at sites of inflammation and infection. Front Immunol 9:754

44. Johnson ED, Schell JC, Rodgers GM (2019) The D-dimer assay. Am J Hematol 94(7):833-839

45. Ferrante $\mathrm{G}$ et al (2020) Risk factors for myocardial injury and death in patients with COVID-19: insights from a cohort study with chest computed tomography. Cardiovasc Res 116(14):2239-2246

46. Shi $S$ et al (2020) Characteristics and clinical significance of myocardial injury in patients with severe coronavirus disease 2019. Eur Heart J 41(22):2070-2079

\section{Publisher's Note}

Springer Nature remains neutral with regard to jurisdictional claims in published maps and institutional affiliations. 